\title{
Supercontinuum Spectrum Engineering in Ge-doped Microstructured Multicore Fiber
}

\author{
D. Modotto ${ }^{1}$, G. Manili ${ }^{1}$, U. Minoni ${ }^{1}$, S. Wabnitz ${ }^{1}$, C. De Angelis ${ }^{1}$, G. Town ${ }^{2}$, A. Tonello ${ }^{3}$, V. Couderc ${ }^{3}$ \\ ${ }^{I}$ Dipartimento di Ingegneria dell'Informazione, Università degli Studi di Brescia, via Branze 38, 25123 Brescia, Italy \\ ${ }^{2}$ Department of Electronic Engineering, Macquarie University, NSW 2109, Australia \\ ${ }^{3}$ XLIM, Université de Limoges, UMR CNRS 6172, 123 Avenue Albert Thomas, 87060 Limoges, France \\ daniele.modotto@ing.unibs.it
}

\begin{abstract}
Supercontinuum generation in a multicore Ge-doped microstructured optical fiber is experimentally studied. The output spectral extension and the presence of four-wave mixing peaks are strongly dependent on the level of doping.

OCIS codes: (190.4370) Nonlinear optics, fibers; (060.4005) Microstructured fibers.
\end{abstract}

\section{Introduction}

Supercontinuum (SC) generation and parametric conversion can be very efficient in a microstructured optical fiber due to the possibility to control its dispersion and to the Kerr nonlinearity of glass. Different phenomena can ignite and contribute to spectral broadening [1,2]: self-phase modulation (SPM), modulation instability (MI), cross-phase modulation (XPM), stimulated Raman scattering (SRS) and four-wave mixing (FWM); the efficiency of these effects is closely connected to the MOF dispersion and particularly to the position of the pump wavelength relative to the zero-dispersion wavelength (ZDW). Since the mode effective index and its dependence on the wavelength is a function of the refractive index difference between the core and the surrounding area, this suggests to control the ZDW by adding dopants [3]. Moreover the Kerr coefficient is almost linearly dependent on Ge concentration and can reach the value $n_{2}=3 \cdot 10^{-16} \mathrm{~cm}^{2} / \mathrm{W}$, which means an increase of $39 \%$ with respect to standard silica glass where $\mathrm{n}_{2}=2.16 \cdot 10^{-16} \mathrm{~cm}^{2} / \mathrm{W}$ [4]. Ge-doped MOFs are well-suited to generate FWM with extremely large Stokes frequency shifts [5]: wavelength conversion from $813 \mathrm{~nm}$ to $1540 \mathrm{~nm}$ with a pump at $1064 \mathrm{~nm}$ was reported in [6], and an efficient FWM process leading to the formation of a Stokes band at $2250 \mathrm{~nm}$ and an anti-Stokes band at $704 \mathrm{~nm}$ was described in [7].

In our work we present a systematic study of SC generation in a multicore microstructured optical fiber (MMOF) where the cores have different levels of doping: therefore, when pumped by the same laser, different cores lead to SC spectra with adjustable spectral features. A MMOF is an ideal testing bench to study the influence of doping since it is relatively simple to move the input pump beam from one core to another without changing the coupling efficiency. Thanks to recent progress in the fabrication of waveguides for coupling light in and out of MMOF [8], we envisage that our multi-spectral source based on MMOFs may provide a key component for realizing a compact SC laser array [9], or a broadband source for spatial mode multiplexed fiber optical communication systems [10].

\section{Microstructured fiber and experimental setup}

In order to study the influence of Ge-doping level on SC generation we consider a MMOF with 7 cores; these cores are not mutually coupled for wavelengths in the visible and near-infrared range.
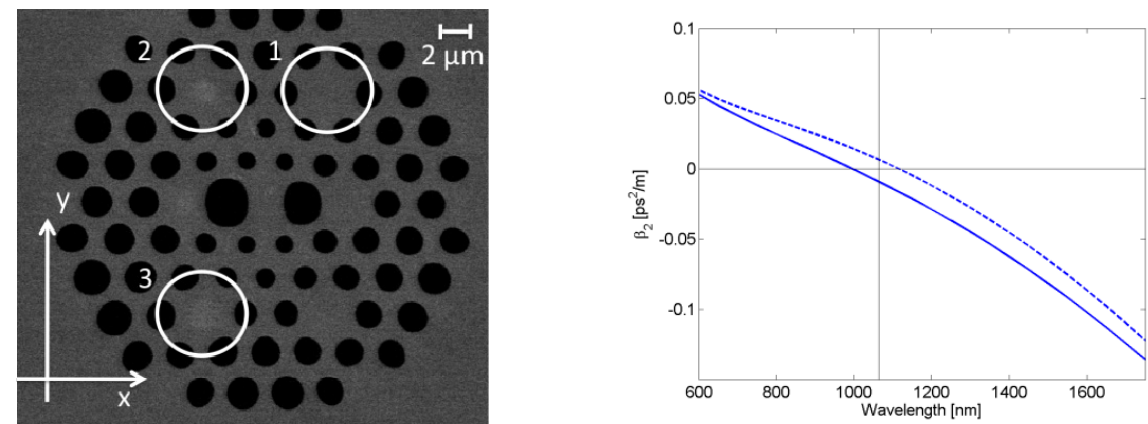

Fig. 1. Left: Scanning electron microscope (SEM) image of the MMOF; the cores under study are circled in white. Right: dispersion curves $\left(\beta_{2}\right)$ for core 1 (continuous line) and core 3 (dashed line); the vertical line corresponds to the wavelength of $1064 \mathrm{~nm}$. 
A SEM image of the MMOF cross section is displayed in Fig.1 (left): it can be observed that the 4 outer cores exhibit the same geometrical structure, whereas core 2 and 3 are Ge-doped. In particular, we estimate a level of doping leading to a maximum refractive index increase of $1.25 \cdot 10^{-2}$ for core 2 and of $2.5 \cdot 10^{-2}$ for core 3 . The 3 cores under study support 2 fundamental modes $\left(\mathrm{LP}_{01}\right.$ like $)$ which are orthogonally polarized: due to the fact that the holes have different radii, the cores are birefringent and their principal axes are oriented at $45^{\circ}$ and at $135^{\circ}$ with respect to the $\mathrm{x}$ axis (see Fig.1 (left) and reference system therein). Fig.1 (right) shows the numerically calculated dispersion curves for $\mathrm{LP}_{01,135^{\circ}}$ in core 1 (undoped) and $\mathrm{LP}_{01,135^{\circ}}$ in core 3 (the one with the highest level of doping); estimated ZDWs for $\mathrm{LP}_{01,135^{\circ}}$ in cores 1,2 and 3 are $996 \mathrm{~nm}, 1043 \mathrm{~nm}$ and $1115 \mathrm{~nm}$, respectively.

In the following section, we report the experimental study of the SC that was observed at the output of a $3 \mathrm{~m}$ long piece of MMOF by using as a pump source a microchip Nd:YAG Q-switched laser [2] working at $1064 \mathrm{~nm}$ and emitting pulses with a duration of $600 \mathrm{ps}$ and an estimated peak power of $6 \mathrm{~kW}$. It must be emphasized that the pump lies in the anomalous dispersion region for core 1 and 2, whereas it is inside the normal dispersion region for core 3. The experimental setup allows us to control both power and polarization of the input beam, whereas a micrometric 3-axis translation stage permits to select in which core light is coupled to. The output power was monitored by means of a broadband power sensor (which also provides a reasonable estimate of the input power, when neglecting MMOF absorption and scattering losses).

\section{Experiments and discussion}

Let us start by experimentally studying SC generation in the undoped core 1. Since the pump at $1064 \mathrm{~nm}$ is in the anomalous dispersion region, by increasing the input power the output spectrum develops MI peaks and broadens towards the long wavelengths side. Fig.2 (left) shows the measured output spectrum for the maximum coupled input power which corresponds to an output peak power $\mathrm{P}_{\text {out }}=1411 \mathrm{~W}$; the residual pump can still be observed and the long tail extends till around $1700 \mathrm{~nm}$. This tail is due to high-order soliton break-up followed by red-shifting induced by intrapulse SRS. In core 1, the output spectrum shows significant energy in the visible region as well.

SC generation leads to a remarkably different spectrum in the low-level doped core 2. During the first stages of propagation the spectrum is enlarged by MI. Next, alongside with the usual spectral broadening in the long wavelength region owing to SRS, nonlinearity also leads to power transfer in the $600-1000 \mathrm{~nm}$ region thanks to group velocity matching between these visible spectral components and the corresponding infrared components. Fig.2 (right) shows the output spectrum for the maximum level of input power (measured $\mathrm{P}_{\text {out }}=1865 \mathrm{~W}$ ): $\mathrm{SC}$ spans from $600 \mathrm{~nm}$ to $1700 \mathrm{~nm}$ with a spectral density that exhibits a $5 \mathrm{~dB}$ flatness on both sides of the pump. No parametric conversion peaks can be observed in the SC spectrum: the only clearly recognizable peak is located at $1094 \mathrm{~nm}$ which represents one of the MI lines of the pump. The MMOF mode when projected on a screen shows an almost circular shape, which provides a clear signature that light is carried by the fundamental $\mathrm{LP}_{01}$ mode, and a vivid red-orange color due to the presence of intense red components in the SC spectrum.
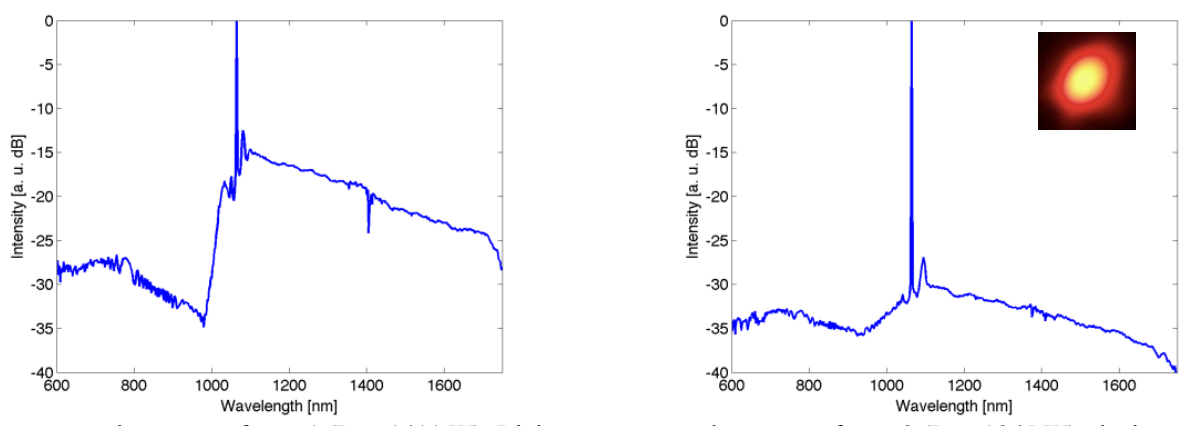

Fig. 2. Left: spectrum at the output of core $1\left(\mathrm{P}_{\text {out }}=1411 \mathrm{~W}\right)$. Right: spectrum at the output of core $2\left(\mathrm{P}_{\text {out }}=1865 \mathrm{~W}\right)$; the inset shows the output projected on a screen.

When we consider the core with the highest doping level (core 3), the SC spectral evolution is markedly different. In fact, even at the lowest levels of power, FWM peaks are visible: for the mode polarized at $135^{\circ}$ the peaks rise at $776 \mathrm{~nm}$ (anti-Stokes) and at $1692 \mathrm{~nm}$ (Stokes); for the mode polarized at $45^{\circ}$ only the anti-Stokes peak at $758 \mathrm{~nm}$ is present in the output spectrum since the corresponding Stokes peak $(1784 \mathrm{~nm})$ is beyond the measuring range of our Ando AQ-6310B spectrum analyzer. Fig.3 (left) shows the output spectrum for an input polarized at $135^{\circ}$ when $\mathrm{P}_{\text {out }}=639 \mathrm{~W}$ (continuous line): the FWM peaks are quite intense $(20 \mathrm{~dB}$ below the pump level). It must be 
emphasized that both Stokes and anti-Stokes signals are carried by the same $\mathrm{LP}_{01}$ mode. The corresponding almost circular modal symmetry makes a core with such level of doping an efficient source of light for coupling into optical fibers at both the red spectral range and in the C-L bands. By further increasing the pump power, the SC spectrum gets wider until it eventually merges with the FWM peaks (broadened by SRS), thus giving rise to a spectrum covering the range 600-1700 nm, as shown in Fig.3 (left) for $\mathrm{P}_{\text {out }}=1407 \mathrm{~W}$ (dashed line).
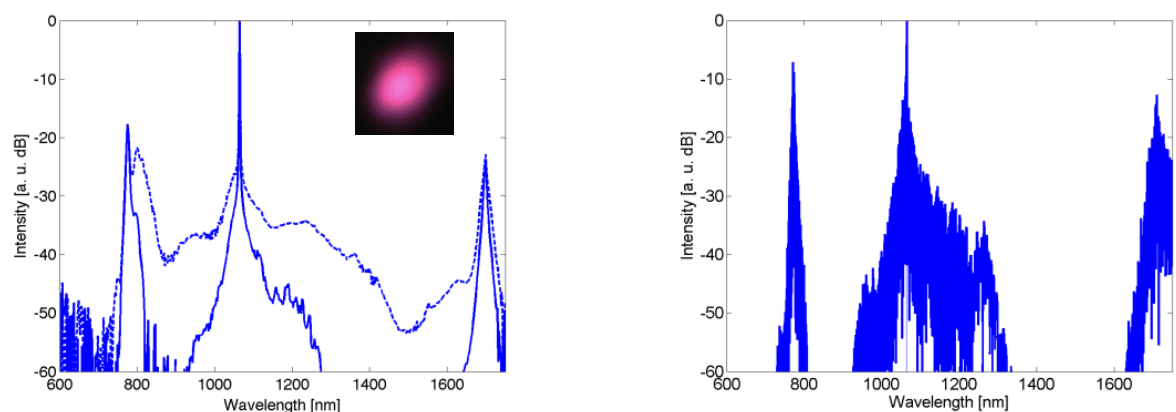

Fig. 3. Left: measured spectra at the output of core $3: \mathrm{P}_{\text {out }}=639 \mathrm{~W}$ (continuous line) and $\mathrm{P}_{\text {out }}=1407 \mathrm{~W}$ (dashed line); the inset shows a photo of the visible $\mathrm{LP}_{01}$ output. Right: calculated spectra at the output of core 3 for an input pulse duration of $60 \mathrm{ps}$ and $\mathrm{P}_{\text {in }}=600 \mathrm{~W}$.

To verify our understanding of the SC development, we carried out extensive numerical solutions of the Generalized Nonlinear Schrödinger Equation [1]. We used the full dispersion curve and included contributions from instantaneous Kerr and delayed Raman effects. It is not practical to simulate the propagation of 600 ps pump pulses, because such temporal width would require an extremely large number of sample points to simulate the spectral broadening. Modelling with 60 ps pulses (i.e. shorter than in experiments, but still in the "CW" regime for SC generation) supports experimental findings. In particular, in Fig.3 (right) we show the numerically calculated spectrum at the output of core 3 for the input polarized at $135^{\circ}$ and with a peak power of $600 \mathrm{~W}$.

\section{Conclusions}

SC spectra obtained at the output of a MMOF may be conveniently engineered by adding a suitable level of germanium in its different cores. Indeed doping not only shifts the ZDW at a value longer or shorter than the pump wavelength, but it also permits for an accurate tuning of their separation. Therefore pumping with a single pump the cores of a doped MMOF leads to SC spectra with different features at its output. Such type of SC source may open new possibilities for versatile engineering of SC spectra: by combining the outputs from these cores it is conceivable to obtain an overall spectral density with extreme spectral coverage and adjustable features.

\section{References}

[1] J.M. Dudley, G. Genty, S. Coen, “Supercontinuum generation in photonic crystal fiber,” Reviews of Modern Physics 78, 1135-1184 (2006).

[2] W.J. Wadsworth, N. Joly, J.C. Knight, T.A. Birks, F. Biancalana, P. St. J. Russell, "Supercontinuum and four-wave mixing with Q-switched pulses in endlessly single-mode photonic crystal fibres," Optics Express 12, 299-309 (2004).

[3] Y.P. Yatsenko, A.D. Pryamikov, V.M. Mashinsky, M.E. Likhachev, A.O. Mavritsky, E.M. Dianov, A.N. Guryanov, V.F. Khopin, M.Y. Salgansky, "Four-wave mixing with large Stokes shifts in heavily Ge-doped silica fibers," Optics Letters 30, 1932-1934 (2005).

[4] Y.P. Yatsenko, A. Mavritsky, "D-scan measurement of nonlinear refractive index in fibers heavily doped with GeO ${ }_{2}$," Optics Letters 32, 3257-3259 (2007).

[5] V. Tombelaine, A. Labruyère, J. Kobelke, K. Schuster, V. Reichel, P. Leproux, V. Couderc, R. Jamier, H. Bartelt, "Nonlinear photonic crystal fiber with a structured multi-component glass core for four-wave mixing and supercontinuum generation," Optics Express 17, 15392-15401 (2009).

[6] Y.P. Yatsenko, A.F. Kosolapov, A.E. Levchenko, S.L. Semjonov, E.M. Dianov, "Broadband wavelength conversion in a germanosilicate-core photonic crystal fiber," Optics Letters 34, 2581-2583 (2009).

[7] A. Labruyère, P. Leproux, V. Couderc, V. Tombelaine, J. Kobelke, K. Schuster, H. Bartelt, S. Hilaire, G. Huss, G. Mélin, "Structured-core $\mathrm{GeO}_{2}$-doped photonic-crystal fibers for parametric and supercontinuum generation," IEEE Photonics Technology Letters 22, 1259-1261 (2010).

[8] R.R. Thomson, H.T. Bookey, N.D. Psaila, A. Fender, S. Campbell, W.N. MacPherson, J.S. Barton, D.T. Reid, A.K. Kar, "Ultrafast-laser inscription of a three dimensional fan-out device for multicore fiber coupling applications," Optics Express 15, 11691-11697 (2007).

[9] L. Li, A. Schülzgen, H. Li, V.L. Temyanko, J.V. Moloney, N. Peyghambarian, "Phase-locked multicore all-fiber lasers: modeling and experimental investigation," Journal of the Optical Society of America B 24, 1721-1728 (2007).

[10] B. Zhu, T.F. Taunay, M.F. Yan, M. Fishteyn, G. Oulundsen, D. Vaidya, "70-Gb/s Multicore Multimode Fiber Transmissions for Optical Data Links," IEEE Photonics Technology Letters 22, 1647-1649 (2010). 